\title{
Caduta accidentale di una paziente sottoposta a riduzione chirurgica di frattura di femore: analisi delle cause profonde
}

\author{
Antonella Barbieri ${ }^{1}$, Giovanna Ferrari ${ }^{2}$ \\ Dirigente Farmacista. SC Qualità e Appropriatezza Prescrittiva, ASL VC (Vercelli). \\ 2 Infermiera. Master di I livello in Management Infermieristico per le Funzioni di Coordinamento-Facoltà di Medicina, Chirurgia e Scienze della Salute - \\ Università del Piemonte Orientale "A. Avogadro" (Novara)
}

\begin{abstract}
Introduction. Inpatient falls in hospital is a relevant problem. The first essential step is to know the risks to prevent them, through reactive or proactive analysis. In this study we used the Root Causes Analysis (RCA) to analyze an accidental fall of a 79-year-old patient after a surgical intervention for a femoral fracture. The aim of the analysis is to evaluate the effect of this method on sanitary professionals.

Material and methods. The study was conducted at the Operative Unity of Orthopaedics and Traumatology, "Beato Matteo Institute", Vigevano. RCA was realised in the following steps: creation of the team, individuation of the causes, collection and evaluation of the information, identification of the solutions and the proposals of improvement.

Results. The RCA showed the following causes: incomplete assessment of the patient, lacking professional skills, lacking of procedures and safety systems, risk factors connected both to the patient and the environment. The improvement proposals were: education and information of professionals, education of patient and caregivers, nursing interventions, environmental strategies and signalling systems.

Discussion. RCA is an useful tool for risk management and it allowed to highlight causes and corrective actions of the accidental fall. Furthermore, this case allowed to underline advantages and disadvantages of this method. Advantages were the involvement of different professionals and sharing of objectives of systems improvement; the importance of the introduction of new instruments for risks identification and specific education to use them. Among the disadvantages we can list: difficulties in finding all the informations to correctly analyse the case, perception of too much bureaucracy in the procedure, and fear of a "punitive" use of the information collected.
\end{abstract}

Keywords: accident falls, falls prevention, Root Cause Analysis, patient safety

Accidental fall of a patient undergone surgical reduction of femoral fracture: Root Causes Analysis Pratica Medica \& Aspetti Legali 2010; 4(1): 21-29

\section{INTRODUZIONE}

Le cadute degli anziani rappresentano una priorità per la sanità pubblica a causa della loro frequenza, del ricorso all'istituzionalizzazione che è associato ai loro esiti, dell'elevata probabilità di causare o aggravare disabilità e dell'elevata mortalità. Le cadute rappresentano la prima causa di incidenti domestici e anche, tra i traumi, la prima causa di ricovero e decesso [1]. È da rilevare come la caduta rappresenti quindi un evento temibile per l'anziano non solo per le conseguenze in termini di disabilità, ma anche per le ripercussioni psicologiche e sociali: la perdita di sicurezza e la paura di cadere possono accelerare il declino funzionale e indurre depressione o isolamento sociale [2].

Si deve pertanto evitare che il paziente subisca ulteriori danni nel momento in cui si rivolge al sistema sanitario per migliorare il suo stato di sa- 
lute [3]. Purtroppo si calcola che l'incidenza delle cadute nelle case di cura e negli ospedali sia 2-3 volte superiore rispetto a quella delle cadute che avvengono nelle abitazioni, e con complicanze maggiori [4].

È per questo che negli Stati Uniti, in molti Paesi europei e da qualche anno anche in Italia è nato il risk management, definito in ambito sanitario "Gestione del Rischio Clinico", che comprende l'insieme delle attività cliniche e amministrative intraprese per identificare, valutare e ridurre i rischi per i pazienti, per lo staff, per i visitatori e i rischi di perdita per l'organizzazione stessa [5].

Il primo passo fondamentale per il risk management è conoscere in modo preventivo i rischi: quali sono gli eventi potenzialmente dannosi, con quale frequenza si possono manifestare e quale impatto possono avere. Senza la conoscenza del rischio non c'è possibilità di preparare o adottare azioni correttive migliorative [6].

Per l'individuazione degli eventi potenzialmente dannosi si usano analisi proattive o reattive. L'analisi proattiva mira all'individuazione e all'eliminazione delle criticità del sistema prima che l'incidente si verifichi; è basata sull'analisi dei processi che costituiscono l'attività e ne individua i punti critici con l'obiettivo di progettare sistemi sicuri.

Lanalisi reattiva prevede uno studio a posteriori degli incidenti ed è mirata a individuare le cause che hanno permesso il loro verificarsi ${ }^{[7]}$.

Tra gli approcci reattivi maggiormente utilizzati troviamo la Root Cause Analysis (RCA), ossia l'analisi delle cause profonde, che costituisce il principale strumento applicabile alla fase di analisi del processo di gestione del rischio, in tutti i settori in cui è attivo un sistema di risk management. Per Root Cause Analysis si intende un'indagine strutturata che ha lo scopo di identificare la causa vera di un problema e le azioni necessarie a eliminarla $[7,8]$.

La RCA è una metodologia applicata allo studio dei fattori causali di un evento avverso, o più in generale di un incidente, che si basa sulla concezione organizzativa dell'errore. L'analisi non si limita a individuare l'errore, ma mira ad analizzare l'intero processo che ha generato l'evento stesso. Lobiettivo principale di questa metodologia è arrivare a comprendere in modo approfondito che cosa è successo, perché è successo e che cosa si può/deve fare per evitare il suo ripetersi $[7,8]$.

L'analisi delle cause profonde deve essere condotta da personale debitamente formato, sia dal punto di vista clinico, sia dal punto di vista metodologico specifico. Un altro carattere rilevante per l'efficacia del metodo è la multidisciplinarietà, con il coinvolgimento prioritario dei soggetti implicati nel processo assistenziale in esame, ma anche di figure con competenze di tipo organizzativo, gestionale e medico-legale. La RCA non può quindi essere condotta da una sola persona, per quanto esperta del problema e delle metodiche. È invece auspicabile che a una sola persona ne sia affidata la responsabilità mediante l'identificazione di un team leader $[7,8]$.

La RCA è efficace solo se è focalizzata sulla risoluzione dei problemi ed è basata sul sistema, sui processi e su metodologie esplicite per evitare bias. È necessario inoltre che tali principi siano completamente condivisi dagli operatori che compiono l'analisi e anche dall'intero sistema $[7,8]$. Le principali tappe dell'analisi possono essere così sintetizzate $[7,8]$ :

- individuazione dei casi: generalmente vengono scelti tra i casi segnalati attraverso il sistema di Incident Reporting, valutando il livello di rischio e l'esito dell'evento stesso;

- raccolta delle informazioni, attraverso l'osservazione diretta (sopralluoghi che permettono di acquisire le possibili cause legate a fattori tecnologici e ambientali), analisi della documentazione clinica (la sua utilità è strettamente correlata all'accuratezza e alla completezza e, spesso, bisogna ricorrere al ricordo degli operatori);

- assemblaggio e valutazione delle informazioni, attraverso particolari schede definite "sequenze degli eventi", che sono da ritenere la base su cui effettuare la vera e propria valutazione multidisciplinare;

- individuazione delle cause, mediante riunioni multidisciplinari, condotte da un facilitatore. È importante richiamare gli obiettivi, i principi alla base della RCA e il fine non punitivo dell'attività. Attraverso una revisione della sequenza degli eventi predisposta e utilizzando gli strumenti opportuni, quali checklist, si giunge all'individuazione delle cause;

- identificazione delle soluzioni, fase in cui si organizzano le idee emerse nella discussione e si trasformano in azioni concrete e immediatamente eseguibili, attraverso un piano che prevede, generalmente, l'azione individuata, i tempi di implementazione e le responsabilità.

Nel presente studio la RCA è stata usata per raccogliere e analizzare le cause profonde alla base di un episodio di caduta accidentale, avvenuto durante il decorso post-operatorio di una paziente sottoposta a riduzione chirurgica di frattura di femore. L'obiettivo generale era valutare l'impatto di tale metodica sugli operatori sanitari della struttura presa in esame, perché la conoscenza di un problema e delle misure da adottare per prevenirlo, permettono all'operatore di lavorare in modo migliore, di individuare le criticità presenti nella realtà operativa e di prendere i necessari provvedimenti, al fine di garantire ai pazienti la sicurezza, che è la più importante dimensione della qualità assistenziale [9]. 


\section{MATERIALI E METODI}

Lo studio è stato condotto nell'Unità Operativa (UO) di Ortopedia-Traumatologia dell'Istituto Clinico Beato Matteo (ICBM) di Vigevano, in cui i pazienti trattati sono generalmente anziani. Lanalisi delle cause e dei fattori contribuenti, che hanno provocato la caduta accidentale, è stata condotta attraverso l'applicazione delle schede di Root Cause Analysis, riportate nel documento del Ministero della Salute "Protocollo per il Monitoraggio degli Eventi Sentinella”, il cui contenuto, per semplicità, è riassunto nel box [10].

La realizzazione della RCA ha previsto le seguenti azioni $[7,8]$ :

- costituzione del team di analisi;

- raccolta delle informazioni;

- assemblaggio delle informazioni;

- analisi delle informazioni e individuazione delle cause;

- identificazione delle soluzioni e delle proposte di miglioramento.

\section{DESCRIZIONE DEL CASO}

Una paziente di sesso femminile (79 anni, peso = $36 \mathrm{~kg}$ ), vedova e pensionata, autosufficiente, con

\section{CAUSE E FATTORI CONTRIBUENTI}

- Ci sono fattori legati all'inquadramento/ assessment del paziente che possono aver contribuito all'evento?

- Ci sono fattori legati alla formazione del personale, o alla competenza del personale che possono avere contribuito all'evento?

- Ci sono strumenti e apparecchiature (o I'uso o il mancato uso di tali strumenti) che possono in qualche maniera avere contribuito all'evento?

- Ci sono fattori legati alla carenza/mancanza di informazione o a errata interpretazione di un'informazione?

- Ci sono fattori legati alla carenza/mancanza di comunicazione?

- Ci sono fattori legati all'applicazione di appropriate politiche, procedure e linee-guida, o alla loro mancanza?

- Ci sono fattori legati al venir meno di sistemi di sicurezza o strumenti con funzione di protezione per il paziente?

- Ci sono fattori legati in maniera specifica al paziente? una lunga storia di accesso ai servizi sanitari locali, viene ricoverata presso l'Unità Operativa (UO) di Ortopedia-Traumatologia per frattura del collo del femore sinistro in varo, di data non recente. Un mese prima del ricovero aveva riportato un trauma da caduta all'anca sinistra, a causa del quale si era recata presso il Pronto Soccorso del nosocomio cittadino dove era stata sottoposta a una radiografia dell'anca sinistra e del bacino, entrambe negative per frattura. Tuttavia la paziente avvertiva un peggioramento della sintomatologia dolorosa con impossibilità alla deambulazione, quindi il medico curante richiedeva un'ulteriore radiografia di controllo dell'anca sinistra, che la signora decideva di eseguire presso la struttura sanitaria in esame e che evidenziava invece la frattura. Il medico radiologo, riscontrata la frattura, contattava l'UO di Ortopedia-Traumatologia per una consulenza specialistica. Il Direttore della UO in questione, visionate le radiografie e visitata la paziente che presentava l'arto sinistro accorciato e dolente alla palpazione e ai movimenti, predisponeva il ricovero allo scopo di sottoporre la signora a intervento chirurgico. La paziente veniva trasferita nella camera di degenza, sistemata nel letto, posizionando l'arto fratturato in una valva, al fine di evitare movimenti azzardati.

Dal giorno del ricovero a quello dell'intervento trascorrevano quattro giorni, durante i quali non si riscontravano particolari problemi; la donna veniva inoltre mobilizzata con arti declivi e seduta a letto con l'aiuto del terapista della riabilitazione.

Il giorno dell'intervento la signora entrava in sala operatoria lucida, tranquilla e collaborante e allo stesso modo rientrava in reparto nel primo pomeriggio. Per tutto il pomeriggio il decorso postoperatorio era stato nella norma. La signora era assistita dal figlio che, dopo cena, faceva rientro al proprio domicilio.

Gli infermieri, al cambio del turno, durante il passaggio delle consegne, non segnalavano problemi di rilievo se non che alla signora era stato infuso un emorecupero di $550 \mathrm{ml}$ e che in serata la temperatura corporea ascellare era di $37,5^{\circ} \mathrm{C}$.

Durante il giro d'ispezione del personale infermieristico del turno notturno la signora, in camera da sola, riposava tranquilla nel letto privo di sponde. Dopo un'ora circa, mentre il marito di un'altra paziente, sottoposta anch'essa a intervento chirurgico nel pomeriggio, si recava presso lo studio infermieristico, passando davanti alla camera della signora, si accorgeva che quest'ultima era riversa sul pavimento. Di conseguenza avvisava il personale infermieristico in turno che, tempestivamente, soccorreva la paziente, trovata a terra in prossimità del letto. Nel cadere, la paziente stessa si autorimuoveva il drenaggio in sede di ferita chirurgica, il catetere venoso e si provocava una ferita lacero-contusa all'altezza dell'arcata sopraccigliare sinistra. 
Il personale infermieristico contattava tempestivamente il medico di guardia in turno che, rilevato l'evento, riposizionava la signora nel letto e apportava le prime cure necessarie del caso: rilevazione dei parametri vitali, sutura della ferita all'altezza dell'arcata sopraccigliare, medicazione in sede di ferita chirurgica, esecuzione di prelievo ematico, posizionamento di sponde al letto.

Venivano contattati telefonicamente il Direttore della UO in questione, in quanto medico reperibile di turno, e il figlio della signora, che decideva di raggiungere la madre per assisterla. La paziente, dopo l'evento, riposava tranquillamente per il resto della notte. L'episodio si è risolto senza danni permanenti né prolungamento della degenza a carico della signora.

\section{RISULTATI: APPLICAZIONE DELLA RCA}

\section{COSTITUZIONE DEL TEAM DI ANALISI}

Nel team di analisi sono stati inseriti tutti i professionisti direttamente coinvolti nell'evento, compatibilmente con le esigenze organizzative della struttura, un rappresentante della direzione sanitaria e un referente del sistema qualità aziendale. Il team leader è stato identificato tra gli operatori della UO dove è avvenuto l'incidente.

\section{RACCOLTA DELLE INFORMAZIONI}

Per cercare di capire come l'incidente sia occorso, sono state raccolte le informazioni attraverso osservazione diretta (sopralluoghi dell'UO), analisi della documentazione clinica (cartella clinica, scheda infermieristica, scheda anestesiologica, procedure) e interviste alle persone coinvolte nell'incidente. L'osservazione diretta ha consentito di acquisire informazioni sul contesto e sull'ambiente in cui l'evento si è verificato e quindi sulle possibili cause legate a fattori tecnologici e ambientali. L'analisi della documentazione clinica è stata la fonte più rilevante di informazioni, anche se non sempre sono stati riportati tutti i passaggi che hanno provocato l'incidente, per individuare i quali si è dovuti ricorrere al ricordo degli operatori.

\section{ASSEMBLAGGIO DELLE INFORMAZIONI}

La fase di sistematizzazione delle informazioni è stata condotta dal team leader (infermiere), che ha riportato le informazioni raccolte in una scheda, specificando, oltre ai dati anagrafici della paziente, il luogo di caduta, l'orario, le modalità, l'attività svolta prima di cadere, la terapia assunta, l'evento associato alla caduta e le conseguenze.

\section{ANALISI DELLE INFORMAZIONI E INDIVIDUAZIONE DELLE CAUSE}

Questa attività, che può essere considerata il cuore della RCA, è stata realizzata rispondendo alle domande riportate nel box riportato a pag 21. La descrizione delle cause e dei fattori contribuenti è sintetizzata in Tabella I. Il contenuto della suddetta tabella è stato analizzato dal team, in modo che tutti i partecipanti concordassero su quanto avvenuto ed eventualmente aggiungessero dettagli sfuggiti in precedenza. Per individuare le cause che hanno determinato l'evento è stato usato il diagramma "a lisca di pesce" (diagramma causaeffetto), riportato in Figura 1.

\section{INDIVIDUAZIONE DELLE SOLUZIONI E DELLE PROPOSTE DI MIGLIORAMENTO}

In questa fase sono state riorganizzate le idee emerse durante la discussione e trasformate in azioni concrete. Pertanto è stato creato un programma contenente gli interventi multidisciplinari da adottare per prevenire il rischio di cadute accidentali. Gli interventi individuati sono stati i seguenti: formazione del personale, valutazione del rischio di caduta, educazione sanitaria al paziente e ai suoi familiari, interventi infermieristici, strategie ambientali.

\section{Formazione del personale}

Lattivazione di interventi formativi deve essere finalizzata ad accrescere le conoscenze sui fattori di rischio di caduta e sulle strategie preventive, non trascurando gli aspetti psicologici derivanti da una caduta (paura di cadere, sicurezza nell'esecuzione delle attività quotidiane) [11].

\section{Valutazione del rischio di caduta}

La valutazione del rischio, metodo efficace per identificare le persone a rischio di caduta, dovrebbe essere effettuata su tutte le persone ammesse in ambienti di cura entro $24-48$ ore e qualora le condizioni generali del paziente si modifichino [12]. Deve essere effettuata mediante l'utilizzo di uno strumento standardizzato (scala di Conley e scala di Stratify) [12] e mediante la valutazione clinica del professionista, che è un elemento irrinunciabile nella definizione del rischio di caduta, in quanto 


\begin{tabular}{|c|c|c|c|}
\hline Cause e fattori contribuenti & Sì & No & Descrizione cause e fattori contribuenti \\
\hline $\begin{array}{l}\text { Ci sono fattori legati } \\
\text { all'inquadramento/assessment } \\
\text { del paziente che possono aver } \\
\text { contribuito all'evento? }\end{array}$ & $x$ & & $\begin{array}{l}\text { Il processo di inquadramento della paziente risulta difettoso nel momento } \\
\text { in cui, non applicando strumenti atti a identificare i pazienti a rischio } \\
\text { di caduta, non si ritiene che la signora sia a rischio di caduta e non } \\
\text { necessiti di interventi di prevenzione particolari (come ad esempio il } \\
\text { posizionamento di sponde al letto e/o di assistenza da parte di qualche } \\
\text { familiare) }\end{array}$ \\
\hline $\begin{array}{l}\text { Ci sono fattori legati alla } \\
\text { formazione del personale, o } \\
\text { alla competenza del personale } \\
\text { che possono avere contribuito } \\
\text { all'evento? }\end{array}$ & $\mathrm{x}$ & & $\begin{array}{l}\text { La mancata valutazione dei pazienti a rischio di caduta, attraverso } \\
\text { I'applicazione di strumenti adatti a prevenirle e ridurle, da parte del } \\
\text { personale infermieristico, segnala una carenza dal punto di vista } \\
\text { metodologico. Tutti i professionisti coinvolti nel processo di cura } \\
\text { hanno seguito un percorso formativo adeguato alla professione che } \\
\text { svolgono, quindi sono dotati di conoscenze e competenze che li rendono } \\
\text { autonomi nel proprio agire. Ė inoltre obbligo di ciascun professionista } \\
\text { I'aggiornamento continuo nel proprio ambito professionale. Le conoscenze } \\
\text { e le competenze acquisite devono essere applicate continuamente nella } \\
\text { pratica clinica per garantire un'adeguata, e sempre migliore, qualità } \\
\text { assistenziale; una carenza/mancanza o inadeguata applicazione di tali } \\
\text { caratteristiche limita l'assistenza erogata ed evidenzia personale non } \\
\text { adeguatamente formato, che viene inteso dall'utente come mancanza di } \\
\text { sicurezza nel sistema sanitario }\end{array}$ \\
\hline $\begin{array}{l}\text { Ci sono strumenti e } \\
\text { apparecchiature (o l'uso o il } \\
\text { mancato uso di tali strumenti) } \\
\text { che possono in qualche maniera } \\
\text { avere contribuito all'evento? }\end{array}$ & $\mathrm{x}$ & & $\begin{array}{l}\text { II letto di degenza della signora non era dotato di sponde (né erano state } \\
\text { applicate separatamente), che avrebbero, probabilmente, impedito il } \\
\text { verificarsi dell'evento }\end{array}$ \\
\hline $\begin{array}{l}\text { Ci sono fattori legati } \\
\text { alla carenza/mancanza } \\
\text { di informazione o ad } \\
\text { errata interpretazione di } \\
\text { un'informazione? }\end{array}$ & & $x$ & \\
\hline $\begin{array}{l}\text { Ci sono fattori legati alla } \\
\text { carenza/mancanza di } \\
\text { comunicazione? }\end{array}$ & & $\mathrm{x}$ & \\
\hline $\begin{array}{l}\text { Ci sono fattori legati } \\
\text { all'applicazione di appropriate } \\
\text { politiche, procedure e linee } \\
\text { guida, o alla loro mancanza? }\end{array}$ & $\mathrm{x}$ & & $\begin{array}{l}\text { Presso I'UO presa in esame non sono presenti linee guida, procedure o } \\
\text { protocolli riguardanti la valutazione del rischio di caduta dei pazienti, la } \\
\text { prevenzione delle cadute, la gestione del paziente caduto in ambiente } \\
\text { ospedaliero, gli interventi/strumenti infermieristici utili a prevenire il } \\
\text { ripetersi di cadute prevenibili nelle strutture sanitarie. Probabilmente } \\
\text { l'esistenza e la relativa applicazione di questi strumenti avrebbe permesso } \\
\text { l'identificazione di fattori di rischio di caduta, con conseguente attuazione } \\
\text { di misure preventive che avrebbero evitato o limitato l'evento }\end{array}$ \\
\hline $\begin{array}{l}\text { Ci sono fattori legati al venir } \\
\text { meno di sistemi di sicurezza } \\
\text { o strumenti con funzione di } \\
\text { protezione per il paziente? }\end{array}$ & $\mathrm{x}$ & & $\begin{array}{l}\text { Il letto di degenza della signora non era dotato si sponde che, fungendo } \\
\text { da ostacolo, I'avrebbero costretta a suonare il campanello di chiamata: } \\
\text { sarebbe potuto così intervenire il personale sanitario evitando l'incidente. } \\
\text { La paziente inoltre era in camera da sola: se si fosse valutata l'opportunità } \\
\text { di metterla in camera con un'altra paziente o di far rimanere il figlio ad } \\
\text { assisterla, si sarebbe magari potuto evitare il fatto }\end{array}$ \\
\hline $\begin{array}{l}\text { Ci sono fattori legati in maniera } \\
\text { specifica al paziente? }\end{array}$ & $\mathrm{X}$ & & $\begin{array}{l}\text { Esistono fattori di rischio legati alla signora che, se presi in } \\
\text { considerazione, avrebbero potuto fungere da campanello d'allarme, } \\
\text { cioè: deficit visivo (nella cartella clinica e nella scheda infermieristica } \\
\text { non era riportato che la paziente era portatrice di occhiali e al momento } \\
\text { della caduta, non li indossava; da ciò ne può derivare una diminuzione } \\
\text { della visione notturna), patologie articolari (la paziente era affetta da } \\
\text { osteoporosi, con conseguenti modificazioni legate all'invecchiamento } \\
\text { dell'apparato muscolo-scheletrico), farmaci (la paziente era stata } \\
\text { sottoposta ad anestesia spinale, con somministrazione di farmaci narcotici } \\
\text { che possono creare effetti quali sedazione e confusione mentale), stato } \\
\text { cachettico (la paziente era in gravi condizioni legate a bisogni nutrizionali } \\
\text { e di disidratazione), età ( } 79 \text { anni), sesso femminile }\end{array}$ \\
\hline
\end{tabular}

Tabella I. Descrizione delle cause e dei fattori contribuenti alla caduta accidentale 


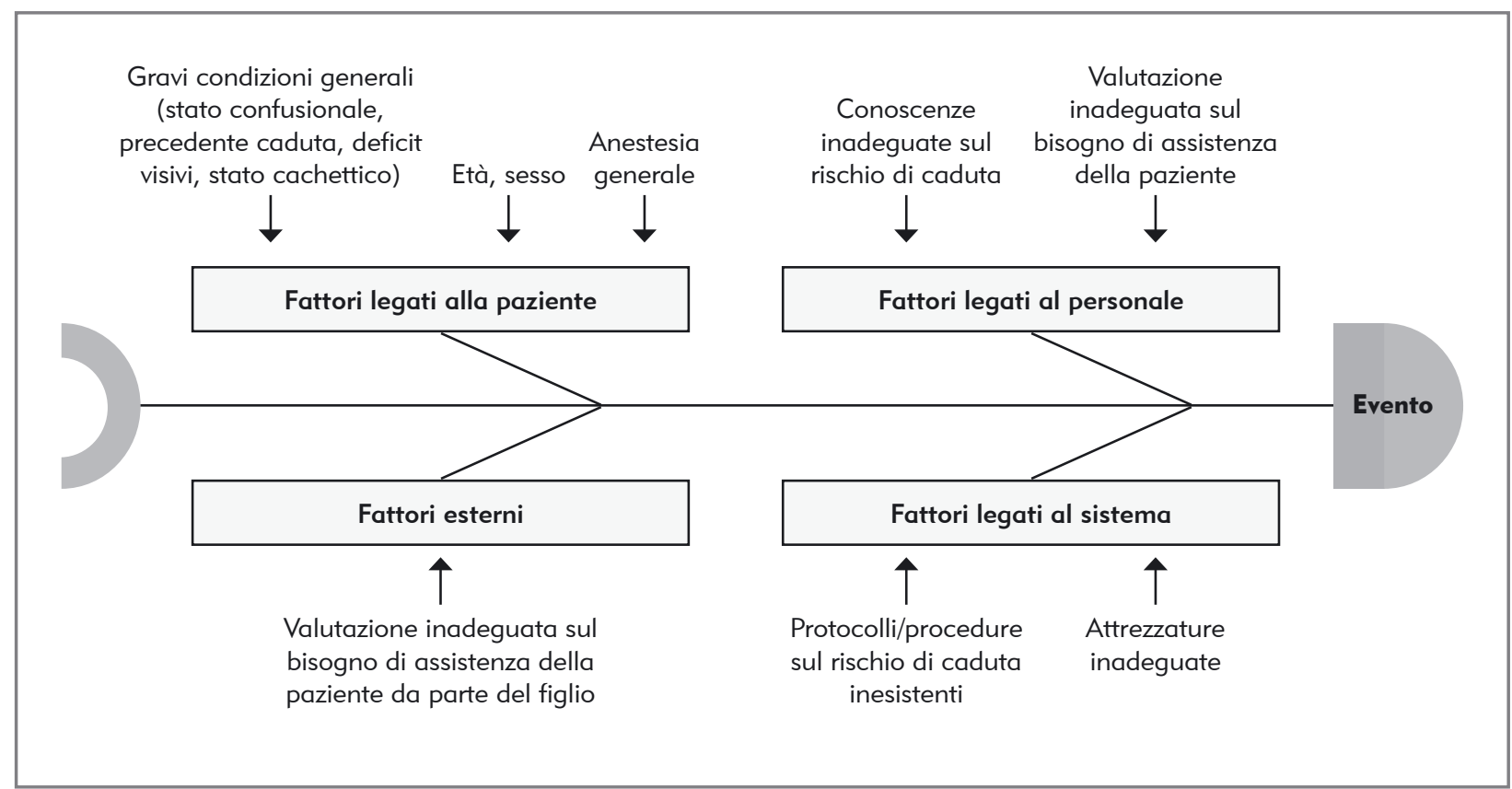

Figura 1. Diagramma "a lisca di pesce"

nessuno strumento di valutazione del rischio, da solo, è in grado di identificare tutte le persone a rischio o tutti i fattori di rischio.

\section{Educazione sanitaria al paziente e ai suoi familiari}

Gli interventi informativi e di educazione sanitaria rivestono notevole importanza e coinvolgono anche il paziente e i familiari, quindi al momento del ricovero si deve [11]: mostrare la stanza di degenza, il bagno, il reparto; mostrare come si suona il campanello di chiamata, sia in camera sia in bagno, verificandone il funzionamento; indicare di informare il personale sanitario ogni qualvolta si vada in bagno o ci si allontani dal reparto; spiegare di effettuare i passaggi posturali in modo lento; distribuire opuscoli divulgativi contenenti programmi formativi per tutti i pazienti nuovi entrati e a rischio di caduta.

\section{Interventi infermieristici}

Le strategie che gli infermieri possono utilizzare per i pazienti a rischio comprendono le azioni descritte in Tabella II [13].

\section{Strategie ambientali}

Per ridurre i rischi ambientali si devono realizzare modifiche delle attrezzature, che siano economiche, ma di alta resa. Pertanto si deve $[11,13]$ : garantire un'adeguata illuminazione per aumentare la visibilità notturna vicino al letto e nel bagno; assicurare l'ordine, evitando di lasciare oggetti sul pavimento e rimuovendo il mobilio, laddove potrebbero costituire ostacolo per i pazienti; stabilizzare i letti, le carrozzine e altro mobilio a uso del paziente; dotare il corridoio, la toilette di maniglie/corrimano a cui aggrapparsi (posti in posizione verticale, piuttosto che orizzontale); identificare e, se possibile, eliminare le caratteristiche ambientali che possono aumentare i potenziali rischi di caduta quali: liquidi sui pavimenti o pavimenti bagnati, pavimenti sconnessi, inadeguata metratura degli ambienti.

\section{Segnalazione}

Le cadute accidentali sono considerate eventi sentinella, che possono provocare gravi danni ai pazienti, pertanto si deve indagare su che cosa è successo e perché è avvenuto. Le informazioni relative all'evento si possono ricavare attraverso le schede di segnalazione di incidente e di descrizione della caduta, che devono essere correttamente e completamente compilate, essendo uno strumento di tutela giuridica e anche di analisi per un possibile cambiamento [14].

\section{DISCUSSIONE}

Lutilizzo della RCA ha consentito di individuare i diversi fattori causali che possono favorire le cadute accidentali, che, nonostante vi siano diversi 


\begin{tabular}{|c|c|}
\hline Aree di intervento & Azioni \\
\hline $\begin{array}{l}\text { Mobilizzazione e } \\
\text { deambulazione }\end{array}$ & $\begin{array}{l}\text { - Controllare che il paziente usi calzature della giusta misura con suole non scivolose } \\
\text { - Controllare che il pannolone, se presente, sia ben posizionato } \\
\text { - Usare presidi/ausili individuali raccomandati, ben progettati e sicuri } \\
\text { - Controllare sedie, poltrone, carrozzine (altezza appropriata per alzarsi e sedersi, dotate di cinture di } \\
\text { sicurezza, di braccioli o maniglie in lattice per prevenire lo scivolamento) }\end{array}$ \\
\hline $\begin{array}{l}\text { Eleminazione } \\
\text { urinaria/fecale }\end{array}$ & $\begin{array}{l}\text { - Controllare i pazienti che assumono lassativi e diuretici } \\
\text { - Seguire i pazienti a rischio durante gli spostamenti e valutare se sono in grado di soddisfare i propri } \\
\text { bisogni } \\
\text { - Verificare periodicamente l'eventuale bisogno di recarsi in bagno, accompagnando il paziente a } \\
\text { intervalli regolari } \\
\text { - Portare e sistemare i pazienti con impellenza vicino ai bagni } \\
\text { - Invitare il paziente a urinare prima del riposo notturno } \\
\text { - Educare i pazienti di sesso maschile, predisposti alle vertigini, a urinare da seduti }\end{array}$ \\
\hline $\begin{array}{l}\text { Stato mentale e } \\
\text { allettamento }\end{array}$ & $\begin{array}{l}\text { - Comunicare in modo rilassato fornendo istruzioni semplici } \\
\text { - Mantenere il letto in posizione bassa, affinché il paziente possa appoggiare i piedi sul pavimento } \\
\text { - Bloccare i freni del letto } \\
\text { - Collocare in prossimità del letto gli oggetti necessari al paziente } \\
\text { - Utilizzare le sponde, magari quelle lunghe metà letto, per ridurre il bisogno del paziente di } \\
\text { scavalcarle per alzarsi dal letto } \\
\text { - Favorire la presenza e il coinvolgimento dei familiari nell'assistenza }\end{array}$ \\
\hline $\begin{array}{l}\text { Terapia } \\
\text { farmacologica }\end{array}$ & $\begin{array}{l}\text { - Limitare l'associazione di farmaci quando è possibile (ad esempio sedativi e analgesici) } \\
\text { - Controllare che il paziente non assuma di sua iniziativa farmaci non prescritti } \\
\text { - Programmare, quando possibile, la terapia infusionale lasciando libero il periodo notturno }\end{array}$ \\
\hline
\end{tabular}

Tabella II. Descrizione degli interventi infermieristici

studi condotti e numerosi articoli pubblicati, continuano a essere un problema di notevole attualità e rilevanza.

Le cause, legate ai pazienti e individuate dalla RCA, coincidono con i dati riportati in letteratura, infatti le cadute dipendono da:

- età: gli anziani (oltre i 65 anni) sono ad aumentato rischio di caduta (la paziente ha 79 anni) [15];

- stato mentale: lo stato mentale deteriorato è il fattore più comunemente identificato nei pazienti che cadono in ospedale. Questo deterioramento è stato anche descritto come confusione o disorientamento, ma anche la carenza di memoria e l'inabilità di comprendere possono aumentare il rischio di caduta del paziente (la paziente, al momento della caduta, era confusa e scarsamente orientata) [13];

- precedenti cadute: le precedenti cadute sono state identificate come fattore associato a un aumentato rischio di caduta. Diversi studi hanno riportato che il $16-52 \%$ dei pazienti può essere vittima di cadute multiple durante la sua ospedalizzazione (la paziente è stata ricoverata per frattura del collo del femore sinistro provocata da una caduta di data non recente, quindi è ricaduta durante la degenza ospedaliera) [13];
- farmaci: i farmaci che agiscono sul SNC del paziente (sedativi, tranquillanti e narcotici) possono aumentare il rischio di cadere (alla paziente, prima dell'intervento chirurgico, sono stati somministrati farmaci narcotici e sedativi) [13];

Infine, i pazienti con più di un fattore di rischio, come nel caso trattato, possono essere più predisposti alle cadute, quanto più aumenta il numero dei fattori di rischio, tanto più cresce il rischio di caduta del paziente [13].

Per quanto concerne i fattori causali legati al personale, la letteratura dimostra che l'identificazione dei pazienti a rischio, attraverso l'uso di strumenti adeguati, consente di realizzare interventi appropriati per ridurre/eliminare le cadute (sulla paziente non è stata eseguita alcuna valutazione perché i suddetti strumenti non sono in uso presso l'UO) [12,16].

Gli strumenti di valutazione del rischio hanno generalmente una buona affidabilità, anche se si deve considerare che, essendo usati da più di un infermiere, possono produrre valutazioni simili del rischio di cadere, ma non sono in grado di distinguere quali pazienti non sono a elevato rischio di caduta [13].

Relativamente all'inadeguata valutazione di posizionamento di sponde al letto, gli studi concordano nel sostenere che la contenzione fisica è un 
metodo controverso per diminuire il rischio di caduta mediante la riduzione di mobilità dei pazienti a rischio, pertanto non può essere avanzata nessuna raccomandazione rispetto all'utilizzo della contenzione e delle sponde $[17,18]$.

La RCA ha evidenziato che ci sono fattori causali legati al sistema, come inadeguata compilazione della documentazione clinica, protocolli/procedure inesistenti o ambigui, mancanza/inadeguatezza di attrezzature o ambienti. La corretta compilazione/tenuta della documentazione clinica esprime la qualità del processo e rappresenta uno dei diritti fondamentali dell'utente. In questa logica trovano giusta collocazione i protocolli e le procedure, sia infermieristici sia medici, intesi come strumento che permette di uniformare i comportamenti degli operatori e di adeguarli alle conoscenze disponibili, contribuendo a garantire un'assistenza corretta, innovativa, basata sulle più recenti acquisizioni tecniche e pratiche, di qualità sicura e personalizzata per il paziente.

Questa esperienza, anche se ancora quantitativamente limitata, ha consentito di mettere in luce vantaggi e svantaggi della metodica.

La RCA ha interessato tutti i professionisti coinvolti nell'evento caduta e ha permesso di condividere gli obiettivi di miglioramento del sistema, ottenendo risposte entusiasmanti di partecipazione legate, probabilmente, alla novità dell'approccio ma anche a un cambiamento culturale. Ha anche stimolato la necessità di introdurre strumenti di segnalazione spontanea degli eventi avversi; infatti l'esperienza ha dimostrato che l'avvio di programmi di analisi degli eventi può di per sé diventare uno stimolo per la segnalazione degli stessi, sia da un punto di vista quantitativo, poiché gli operatori sono stimolati a segnalare se sono consapevoli che il loro lavoro sarà portato all'attenzione di chi è in grado di attivare azioni di miglioramento, sia da un punto di vista qualitativo, in quanto la comprensione dell'utilizzo delle segnalazioni (analisi e trattamento del rischio) induce a focalizzarsi sugli eventi realmente rilevanti.

Ulteriori considerazioni possono essere fatte sugli specifici strumenti utilizzati e illustrati in precedenza, che sembrano essere particolarmente appropriati nell'ambito della RCA. Consentono infatti di esplorare le cause di un evento sia valutando le diverse tipologie di fattori coinvolti (umani, tecnologici, strutturali, organizzativi), sia il livello organizzativo in cui si collocano (di operatore, di ambiente lavorativo, di organizzazione e strategia). Permettono inoltre di effettuare già una classificazione delle cause, anche se è necessario proporli con alcune accortezze: linguaggio familiare alla realtà clinica, terminologia il più possibile univoca (eventualmente corredata di esempi) e classificazione esaustiva. A tal proposito è utile sottolineare che è necessaria una formazione specifica all'uso degli strumenti da parte degli operatori, a cui si può sopperire con la presenza di un esperto, ed è indispensabile che tutti gli operatori coinvolti siano formati in modo approfondito sugli obiettivi e sui principi alla base della RCA. Solo questa formazione preliminare e un richiamo costante alla necessità di non fermarsi alle cause prossime, di non colpevolizzare persone o funzioni, di rimanere focalizzati sul sistema e sulla sicurezza dello stesso, consentono di ottenere i risultati auspicati.

Un ulteriore elemento per garantire l'efficacia della RCA è che tutte le cause individuate siano prese in esame e classificate in base alla rilevanza e alla risolvibilità. L'output dell'indagine dovrà infatti essere un rapporto il più possibile strutturato, da diffondere a tutti i professionisti interessati, che contenga sia l'elenco e la descrizione delle cause individuate, ma anche chiare indicazioni su possibili strategie correttive da implementare.

Per quanto riguarda, invece, le criticità rilevate va segnalata una certa difficoltà nel reperimento di tutte le informazioni utili alla valutazione dell'evento, nonostante l'utilizzo integrato delle fonti informative. Uno dei problemi maggiori è stato sicuramente l'impegno in termini di tempo e di risorse umane necessari per sviluppare un intero processo di RCA; è tuttavia presumibile che una maggiore esperienza possa portare a un utilizzo più fluido dello strumento, da applicare solo ai casi selezionati per rilevanza e significatività.

Un altro elemento critico è stata la percezione da parte dei professionisti di un'eccessiva burocratizzazione della procedura e un'iniziale diffidenza da parte degli operatori coinvolti, derivata probabilmente dalla paura di un utilizzo delle informazioni a scopo punitivo. Successivamente, però, grazie anche all'utilizzo degli strumenti della RCA, si è notato un maggiore coinvolgimento e partecipazione da parte dei professionisti che non conoscevano queste tecniche.

In conclusione si può affermare che la RCA si è rivelata un potente strumento di cambiamento culturale, accettato da tutti, perché ha l'indubbio vantaggio di avvicinarsi di più al linguaggio dei professionisti sanitari e di assicurare una loro perfetta integrazione, al fine di garantire la sicurezza dei pazienti fruitori dei servizi sanitari [9].

\section{DISCLOSURE}

Gli Autori dichiarano di non avere alcun confitto di interessi di natura finanziaria. 


\section{BIBLIOGRAFIA}

1. Pitidis A, Giustini M, Taggi F. La sorveglianza degli incidenti domestici in Italia. Roma: ISS, 2005

2. Registered Nurses' Association of Ontario. Prevention of falls and fall injures in the older adult. Toronto: Registered Nurses' Association of Ontario, 2005

3. Sage WB. Medical liability and patient safety. Health Aff 2003; 22: 27-36

4. PNLG 13. Prevenzione delle cadute da incidente domestico negli anziani maggio 2009. Disponibile su: http://www.ministerosalute.it/imgs/C_17_pubblicazioni_975_allegato.pdf

5. Joint Commission on Accreditation of Healthcare Organizations (JCAHO). http://www.jcaho.org

6. Ministero della Salute. Commissione tecnica sul Rischio Clinico (DM 5 marzo 2003). Il Risk Management in Sanità il problema degli errori. Roma: Ministero della Salute, 2004

7. Ministero della Salute. Dipartimento della Qualità Direzione Generale della Programmazione Sanitaria, dei Livelli di Assistenza e dei Principi Etici di Sistema Ufficio III. Sicurezza dei pazienti e gestione del rischio clinico: Manuale per la formazione degli operatori sanitari. Disponibile su: http://www.ministerosalute.it/imgs/C_17_pubblicazioni_640_allegato.pdf

8. Agenzia Sanitaria Regionale dell'Emilia Romagna. La Root Cause Analysis per l'analisi del rischio nelle strutture sanitarie. Sussidi per la gestione del rischio 6. Bologna: Agenzia Sanitaria Regionale dell'Emilia Romagna, 2006

9. Department of Health. Building a safer NHS for patients. London: The Stationery Office, 2001

10. Ministero della Salute. Dipartimento della Qualità Direzione Generale della Programmazione Sanitaria, dei Livelli di Assistenza e dei Principi Etici di Sistema Ufficio III. Osservatorio Nazionale sugli Eventi Sentinella Protocollo per il Monitoraggio degli Eventi Sentinella. Roma: Ministero della Salute, 2009

11. Azienda Ospedaliera di Bologna Policlinico S. Orsola Malpighi. Suggerimenti di pratica clinica per la prevenzione delle cadute dei pazienti in ospedale. Bologna: Azienda Ospedaliera di Bologna, 2004

12. Chiari P, Mosci D, Fontana S. Valutazione di due strumenti di misura del rischio di cadute dei pazienti. Assist Inferm Ric 2002; 21: $117-24$

13. Mosci D. Cadute in ospedale. Best Practice 1998; 2

14. Vanzetta M, Vallicella F. Qualità dell'assistenza, indicatori, eventi sentinella: le cadute accidentali in ospedale. Management Infermieristico 2001; 2: 32-6

15. Rubenstein LZ. Falls in older people: epidemiology, risk factors and strategies for prevention. Age Ageing 2006; 35(S2): 37-41

16. Haines TP, Bennell KL, Osborne RH, Hill KD. Effectiveness of targeted falls prevention programme in subacute hospital setting: randomised controlled trial. BMJ 2004; 328: 1-6

17. Veterani B, Urbini D, Carfagna M, Aguzzoni N. Prove di efficacia sulla contenzione fisica nella riduzione delle cadute in ospedale. Centro Studi EBP AUSL Cesena. Servizio Sanitario Regionale Emilia Romagna

18. Zuccone N. I mezzi di contenzione. In: Nardi R, Lince M.P. Il Risk Management infermieristico in Medicina Interna. Torino: Centro Scientifico Editore, 2004

\section{CORRESPONDING AUTHOR}

Dott.ssa Antonietta Barbieri; e-mail: antonietta.barbieri@med.unipmn.it 